\title{
WUNED Research

\section{Correlación entre factores de riesgo de enfermedad cardiovascular en 1084 parejas de cónyuges costarricenses. El Proyecto CRELES-RC}

\author{
Eduardo Aguilar Fernández ${ }^{1}$ (D) \& Ana Maricela Carballo Alfaro ${ }^{2}$ (D)
}

1. Universidad Nacional, Facultad de Ciencias Exactas y Naturales, Escuela de Matemática, Heredia, Costa Rica; eduardo.aguilar.fernandez@una.cr

2. Hospital Nacional de Geriatría y Gerontología, Departamento de Farmacia, San José, Costa Rica; anamaricelac@hotmail.com

Recibido 28-IV-2020 • Corregido 01-IX-2020 • Aceptado 29-IX-2020

DOI: https://doi.org/10.22458/urj.v12i2.3106

\begin{abstract}
Correlation among risk factors for cardiovascular disease in 1084 Costa Rican couples. The CRELES-RC project." Introduction: Marital environment can contribute to similarities in lifestyle and morbidity between spouses, as they share common life habits and health risks. Objective: To investigate concordance between various risk factors for cardiovascular disease in couples of Costa Rican spouses. Methods: We analyzed 1084 couples from the Costa Rican Longevity and Healthy Aging Study. We used Pearson's correlation coefficients, and Multivariate within-pair correlations obtained by analyzing dyadic data in the context of the Actor-Partner Interdependence Model (APIM). To estimate the APIM we used structural equation models, and logistic regression analyses to calculate the odds ratio of a spouse's presenting one risk factor on the basis of the other spouse's risk factor status. Results: After adjustment for age, smoking and physical activity, the strongest spousal concordance was for body mass index $(r=0,108,95 \%$ confidence interval: $0,04-0,14)$ and the lowest for $C$ reactive protein $(r=0,067: 0,01-0,16)$. People whose spouse has diabetes, hypertension, central adiposity, high body mass index or inflammation are more likely to have the same disease. The adjusted odds ratios were 2,837 (95\% confidence interval: $2,02-3,98)$ for diabetes; 1,357 $(1,06-1,74)$ for hypertension; $1,508(1,08-2,10)$ for central adiposity; $1,777(1,25-2,53)$ for high body mass index; and $1,357(1,02-1,80)$ for inflammation. Conclusions: There is a small, but significant, spousal concordance between the different risk factors for cardiovascular disease.
\end{abstract}

Keywords: sex, spouse, risk factor, cardiovascular disease, spousal concordance.
RESUMEN. Introducción: El ambiente marital compartido puede contribuir a similitudes en el estilo de vida y la morbilidad entre cónyuges, ya que comparten hábitos de vida y riesgos de salud comunes. Objetivo: Investigar la concordancia entre factores de riesgo cardiovascular en cónyuges costarricenses. Métodos: Analizamos 1084 parejas del proyecto Costa Rica: Estudio de Longevidad y Envejecimiento Saludable (CRELES-RC). Calculamos coeficientes de Pearson y modelos de ecuaciones estructurales del Actor Partner Interdependence Model para estimar correlaciones entre factores de riesgo. Estimamos modelos de regresión logística para el cociente de posibilidades de que un cónyuge presente un factor de riesgo según ese factor en el otro cónyuge. Resultados: Tras ajustar por edad, tabaquismo y actividad física, la correlación más fuerte se dio en el índice de masa corporal ( $r=0,108$; intervalo de confianza del 95\%: 0,04 $0,14)$, y el menor con la proteína C reactiva $(r=0,067: 0,01$ $-0,16)$. Las personas cuyo cónyuge padece de diabetes, hipertensión, adiposidad central, elevado índice de masa corporal o inflamación, tienen mayor probabilidad de presentar la misma enfermedad. Los cocientes ajustados fueron 2,837 (intervalo de confianza del 95\%: 2,02 - 3,98) para diabetes, $1,357(1,06-1,74)$ para hipertensión, 1,508 $(1,08-2,10)$ para adiposidad central, $1,777(1,25-2,53)$ para elevado índice de masa corporal y 1,357 $(1,02-1,80)$ para inflamación. Conclusiones: Existe concordancia conyugal significativa, aunque pequeña, entre los factores de riesgo de enfermedad cardiovascular.

Palabras clave: sexo, cónyuge, factor de riesgo, enfermedad cardiovascular, concordancia conyugal. 
El ambiente marital compartido puede contribuir a similitudes en el estilo de vida y la morbilidad entre cónyuges, ya que comparten hábitos de vida y riesgos de salud comunes (Jurj et al., 2006). La concordancia conyugal busca describir el impacto del estilo de vida compartido y el entorno socioeconómico, independientemente de las influencias genéticas (Lee et al., 2011). Si existen evidencias de concordancia conyugal para los factores de riesgo cardiovasculares, el entorno familiar compartido puede convertirse en una amenaza para los cónyuges de pacientes que tienen un alto riesgo de desarrollar una enfermedad (Di Castelnuovo, Quacquaruccio, Donati, de Gaetano, \& lacoviello, 2009).

Por ejemplo, se ha mencionado que el tabaquismo conyugal está relacionado con accidentes cerebrovasculares (Qureshi, Fareed, Suri, Kirmani, \& Divani, 2005). En el caso de la enfermedad cardiovascular, estudios previos han determinado la presencia de correlaciones entre factores de riesgo tradicionales como la presión sanguínea, el colesterol y el índice de masa corporal (Jee, Suh, Won, \& Kim, 2002; Di Castelnuovo et al., 2007; Lee et al., 2011) o incluso el colesterol HDL, triglicéridos, la circunferencia de la cintura y la glucosa (Di Castelnuovo et al., 2007; Lee et al., 2011). También se han hallado correlaciones importantes en el caso de otros factores que estudios recientes han asociado con enfermedad cardiovascular, relacionados con la inflamación como el caso de la proteína $\mathrm{C}$ reactiva (PCR) y el sistema de coagulación como el factor VII (FVII) (Di Castelnuovo et al., 2007).

Por otro lado, se ha hallado concordancia conyugal en el síndrome metabólico (SM), lo que ha llevado a la conclusión de que factores ambientales compartidos contribuyen al desarrollo del SM (Kim et al., 2006; Lee et al., 2011).

Además, se ha determinado que el riesgo coronario a los 10 años se correlaciona fuertemente entre cónyuges, lo que indica que las parejas además de compartir hábitos de estilo de vida y factores de riesgo metabólico comunes y nuevos, también comparten el riesgo global de predicción de eventos cardiovasculares (Di Castelnuovo et al., 2007).

Partiendo del supuesto que los cónyuges no están relacionados genéticamente, las asociaciones entre cónyuges que han sido observadas sugieren que su entorno compartido es el principal contribuyente a resultados de salud similares (McAdams et al., 2011).

El objetivo del estudio fue investigar el grado de concordancia entre diversos factores de riesgo metabólicos de la enfermedad cardiovascular en parejas de cónyuges costarricenses participantes en el estudio CRELES-RC.

\section{MATERIALES Y MÉTODOS}

Población de estudio: CRELES-RC: Costa Rica: Estudio de Longevidad y Envejecimiento Saludable contempla una nueva cohorte de jubilación nacida entre 1945 y 1955 en la que se realizaron 2798 entrevistas entre enero de 2010 y diciembre de 2011. También se entrevistaron 1395 cónyuges coresidentes independientemente de su año de nacimiento. Este proyecto fue desarrollado por la Universidad de California en Berkeley y el Centro Centroamericano de Población (CCP) de la Universidad de Costa Rica con fondos del Instituto Nacional sobre el Envejecimiento de los Estados Unidos (subvención NIH R01 AG031716). Detalles relacionados con la aprobación, financiamiento, consideraciones éticas, diseño del estudio y marco muestral, entre otros aspectos, han sido descritos previamente (Dow, Brenes, \& Rosero, 2013). Para la presente investigación se excluyeron todas aquellas personas participantes cuyos cónyuges no fueron entrevistados, así como todos aquellos casos que tuvieran valores faltantes. De esta manera, se consideraron 1084 parejas de cónyuges. 
Variables de estudio: En CRELES-RC, información general de la población como la edad, sexo, zona de residencia, área de residencia, nivel educativo, condición de fumado, actividad física fueron obtenidas a través de las entrevistas. La presión arterial (en mmHg: milímetros de mercurio) se midió dos veces durante la entrevista principal, con un intervalo de tiempo promedio de 20 minutos entre cada una. En esta investigación se consideró la media aritmética entre las dos mediciones como valor final de la presión arterial. Otros detalles sobre la medición de la presión arterial, la circunferencia de la cintura (CC) y el peso han sido descritas previamente (Dow et al., 2013; Aguilar \& Carballo, 2020). El índice de masa corporal (IMC) se determinó como el cociente entre el peso (en $\mathrm{kg}$ ) y la altura $\left(\mathrm{en}^{2}\right.$ ). Se consideraron los biomarcardores como el colesterol total (CT), el C-HDL, la HbA1c y la PCR para los cuales CRELES-RC estableció los respectivos protocolos (Dow et al., 2013). Para la presente investigación el IMC se clasificó en dos categorías (IMC < 25 e $I M C \geq 25$ ). La adiposidad central (AC) fue definida si $C C \geq 102 \mathrm{~cm}$ en hombres y $C C \geq 89 \mathrm{~cm}$ en mujeres, hipertensión fue definida si PS $\geq 140 \mathrm{mmHg}$ o $P D \geq 90 \mathrm{mmHg}$ en el valor promedio de las mediciones, el colesterol alto fue definido si CT $\geq 200 \mathrm{mg} / \mathrm{dl}$ y bajo colesterol HDL se definió si C-HDL $\leq 40 \mathrm{mg} / \mathrm{dl}$ en hombres y C-HDL $\leq 50 \mathrm{mg} / \mathrm{dl}$ en mujeres (Smith et al., 2011). Diabetes mellitus Tipo 2 (diabetes) fue definida si $\mathrm{HbA1c} \geq 6,5 \%$ (American Diabetes Association, 2018). La inflamación fue definida si $\mathrm{PCR}>3 \mathrm{mg} / \mathrm{l}$, pues se menciona que la presencia de estos niveles son indicadores de alto riesgo de enfermedad cardiovascular (Pearson et al., 2003). La condición de persona fumadora (tabaquismo) se consideró en dos categorías: no fumadora (si nunca ha fumado cigarrillos) y fumadora (si la persona indicó fumar de forma regular). La actividad física se clasificó en dos categorías (realiza o no realiza actividad física rigurosa durante el último año considerando el momento de la entrevista).

Análisis de los datos: Los datos se describen como promedio y desviación estándar (DE) en el caso de variables continuas y como cantidad y porcentaje para variables categóricas. Para la PCR se utilizó la mediana y el rango intercuartílico (RI). Se utilizó la prueba de suma de rangos de Wilcoxon para el análisis estadístico de las variables continuas. En el caso de las variables categóricas, se utilizó la prueba chi cuadrado.

Se utilizó el coeficiente de correlación de Pearson para estimar las correlaciones univariadas entre cónyuges a nivel general como por estrato. La estatificacón se llevó a cabo por zona (urbana o rural) y área (área metropolitana: GAM u otra) de residencia así como por nivel educativo ( $\leq 60>6$ años de escolaridad). Con el fin de establecer las diferencias entre las correlaciones de los cónyuges por estrato se determinó el coeficiente $q$ de Cohen como medida de tamaño de efecto (Cohen, 1988). Se calcularon correlaciones multivariadas entre los cónyuges analizando datos diádicos en el contexto del Actor-Partner Interdependence Model (APIM), el cual es utilizado para medir la influencia que los miembros de la pareja (díada) tienen entre sí (Fitzpatrick, Gareau, Lafontaine, \& Gaudreau, 2016). En la estimación del APIM se utilizaron modelos de ecuaciones estructurales, las cuales tratan los datos de los grupos de manera anidada dentro de la pareja, lo que permite que en las investigaciones sea posible examinar la influencia que las personas de las parejas ejercen sobre sí mismos y sobre la otra persona (Cook \& Kenny, 2005).

Luego se utilizaron modelos de regresión logística para calcular la razón de posibilidades (OR) de que un cónyuge presente un factor de riesgo sobre la base del estado de dicho factor de riesgo en el otro cónyuge. Estas asociaciones se probaron en modelos separados, tanto si se consideraba el estado del factor de riesgo del esposo o el de la esposa como variable respuesta. En cada caso se construyó un modelo univariado y dos modelos multivariados, uno que consideraba dentro de las variables independientes factores de riesgo del esposo y otro que consideraba los factores de riesgo de la esposa. Las variables consideradas en los modelos multivariados son la edad, la actividad física y el tabaquismo. En el caso de los modelos en los que la variable respuesta era continua, los factores de riesgo CT, C-HDL, PS, PD, CC, IMC y PCR se consideraron en su forma 
continua. En el caso de los modelos con variable respuesta binaria, los factores de riesgo se consideraron en su forma binaria (colesterol alto, bajo C-HDL, diabetes, hipertensión, IMC superior al normal, AC e inflamación). En los distintos modelos la edad fue considerada en su forma continua; el tabaquismo y la actividad física en su forma categórica. Dado que la estimación de las asociaciones se realizaron por separado para esposos y esposas, se evalúo la simetría de la asociación, es decir, verificar si el efecto del estado del factor de riesgo del esposo en la esposa era el mismo que provocaba el estado de dicho factor de la esposa en el esposo (McAdams et al., 2011).

Los análisis estadísticos univariados y los modelos de regresión logísticas se llevaron a cabo utilizando el programa STATA versión 13.1 (stat Corp. USA). En el caso de la estimación del APIM se utilizó la aplicación Giving Dyadic Data Analysis Away (Stas, Kenny, Mayer, \& Loeys, 2018). Los valores $p<0,05$ se consideraron estadísticamente significativos.

\section{RESULTADOS}

De las parejas en estudio, el $51,8 \%$ vive en zona urbana y el $47,6 \%$ reside en la gran área metropolitana. En cuanto al nivel educativo, la mayor propoción de personas cuenta con nivel de primaria, $61,6 \%$ en hombres y $63,7 \%$ de las mujeres. El peso y la altura promedio son $74,8 \mathrm{~kg}$ y $1,66 \mathrm{~m}$ en hombres y $70,3 \mathrm{~kg}$ y $1,54 \mathrm{~m}$ en mujeres, respectivamente (Cuadro 1 ).

\section{CUADRO 1}

Costa Rica. Características generales de las parejas participantes en el proyecto CRELES-RC. ( $\mathrm{n}=1084$ parejas)

\begin{tabular}{lccc}
\hline \multicolumn{1}{c}{ Característica } & Hombre (\%) & Mujer (\%) & $p$ \\
\hline Zona & & & 0,470 \\
Urbana & 51,8 & 51,8 & \\
Rural & 48,2 & 48,2 & \\
Área, \% & & & 0,092 \\
GAM & 47,6 & 47,6 & \\
Otra & 52,4 & 52,4 & \\
Nivel educativo & & & 0,007 \\
Ninguno & 8,3 & 6,1 & \\
Primaria & 61,6 & 63,7 & \\
Secundaria & 16,5 & 19,9 & \\
Superior & 13,6 & 10,3 & \\
Peso en kg, (DE) & $74,8(14,1)$ & $70,3(13,7)$ & $<0,001$ \\
Altura en m, (DE) & $1,66(0,07)$ & $1,54(0,06)$ & $<0,001$ \\
\hline
\end{tabular}

Fuente: CRELES-RC. Año 2011

Las comparaciones entre cónyuges reflejan niveles más elevados en los factores de riesgo en las mujeres con excepción de la presión sistólica y la CC; sin embargo, las diferencias no son significativas ( $p=0,156$ y $p=0,140$, respectivamente). Los hombres son más propensos a fumar y realizar actividad física. Los porcentajes de personas diabéticas e hipertensas son muy similares en ambos sexos ( $p=0,063$ y $p=0,385$, respectivamente), mientras que la proporción de mujeres con 
presencia de niveles de PCR superiores a $3 \mathrm{mg} / \mathrm{l}$ es casi el doble que la de hombres (42,3\% vs $24,5 \%$ ). Una alta proporción de mujeres y hombres presentan mediciones del IMC $\geq 25 \mathrm{~kg} / \mathrm{m}^{2}$ (Cuadro 2 ).

\section{CUADRO 2}

Costa Rica. Factores de riesgo cardiovascular de las parejas participantes en el proyecto CRELES-RC. ( $\mathrm{n}=1084$ parejas)

\begin{tabular}{|c|c|c|c|}
\hline & \multicolumn{2}{|c|}{ Sexo } & \multirow{2}{*}{$p$} \\
\hline & Hombre & Mujer & \\
\hline Edad (DE) & $61,9(6,4)$ & $56,3(6,8)$ & $<0,001$ \\
\hline CT en mg/dl (DE) & $193,7(38,6)$ & $205,8(40,0)$ & $<0,001$ \\
\hline C-HDL en mg/dl (DE) & $37,2(11,3)$ & $43,4(12,2)$ & $<0,001$ \\
\hline HbA1c en \% (DE) & $6,1(1,1)$ & $6,2(1,2)$ & $<0,001$ \\
\hline PS en mmHg (DE) & $138,0(19,5)$ & $136,9(19,6)$ & 0,156 \\
\hline PD en mmHg (DE) & $79,5(11,2)$ & $80,5(10,4)$ & 0,008 \\
\hline$C C$ en $\mathrm{cm}(\mathrm{DE})$ & $96,5(11,5)$ & $97,5(13,2)$ & 0,140 \\
\hline IMC en $\mathrm{kg} / \mathrm{m}^{2}(\mathrm{DE})$ & $27,1(4,5)$ & $29,7(5,4)$ & $<0,001$ \\
\hline PCR en mg/l (RI) & $2,0(1-3)$ & $3,0(2-6)$ & $<0,001$ \\
\hline Tabaquismo, \% & 22,7 & 7,4 & $<0,001$ \\
\hline Actividad física, \% & 45,3 & 18,5 & $<0,001$ \\
\hline Colesterol alto, $\%$ & 42,2 & 55,3 & $<0,001$ \\
\hline Bajo C-HDL, \% & 68,8 & 76,6 & $<0,001$ \\
\hline Diabetes, \% & 24,4 & 27,9 & 0,063 \\
\hline Hipertensión, \% & 41,9 & 43,7 & 0,385 \\
\hline$A C, \%$ & 27,9 & 75,9 & $<0,001$ \\
\hline IMC, \% & 71,7 & 84,6 & $<0,001$ \\
\hline Inflamación, \% & 24,5 & 42,3 & $<0,001$ \\
\hline
\end{tabular}

Fuente: CRELES-RC. Año 2011

Para la edad se obtuvo un coeficiente de correlación de 0,41 ( $p<0,0001)$. Los diferentes factores de riesgo metabólicos antropométricos se correlacionaron entre las parejas en el análisis univariado. Estas correlaciones variaron entre 0,067 y 0,110. Todas las asociaciones resultaron significativas y la correlación más fuerte se dio en el IMC. En el análisis multivariado se obtienen intervalos de confianza cuyos límites inferiores son mayores a cero luego de ajustar por edad (modelo 1) o edad, tabaquismo y actividad física (modelo 2). La correlación más fuerte en el análisis multivariado $(r=0,112$, IC95\%: 0,06-0,21) se volvió a presentar en el IMC. La fuerza en la asociación de los factores no mostró variaciones importantes en los distintos análisis (Cuadro 3).

Las correlaciones por estrato presentan valores para la $q$ de Cohen inferiores a 0,15. A excepción del colesterol total $(q=0,13)$ y la $\operatorname{HbA1c}(q=0,12)$ donde se refleja un efecto pequeño, para el resto de los factores de riesgo el valor de $q$ inferior a 0,10 es un indicador de un efecto no relevante (Cohen, 1988) (Cuadro 4). 


\section{CUADRO 3}

Costa Rica. Correlación univariada y multivariada de los distintos factores de riesgo de enfermedad cadiovascular en las parejas participantes en el proyecto CRELES-RC. ( $n=1084$ parejas)

\begin{tabular}{ccccccc}
\hline \multirow{2}{*}{ Factor } & \multicolumn{2}{c}{ Univariado } & \multicolumn{2}{c}{ Modelo 1 } & \multicolumn{2}{c}{ Modelo 2 } \\
& $\boldsymbol{r}$ & $\boldsymbol{p}$ & $\boldsymbol{r}$ & IC 95\% & $\boldsymbol{r}$ & IC 95\% \\
\hline CT & 0,099 & 0,001 & 0,095 & $0,04-0,16$ & 0,091 & $0,03-0,15$ \\
C-HDL & 0,076 & 0,013 & 0,075 & $0,01-0,15$ & 0,077 & $0,02-0,13$ \\
HbA1C & 0,093 & 0,002 & 0,093 & $0,03-0,16$ & 0,096 & $0,04-0,15$ \\
PS & 0,067 & 0,008 & 0,077 & $0,02-0,13$ & 0,082 & $0,02-0,15$ \\
PD & 0,093 & 0,002 & 0,091 & $0,03-0,14$ & 0,096 & $0,04-0,17$ \\
CC & 0,073 & 0,016 & 0,080 & $0,02-0,16$ & 0,087 & $0,02-0,13$ \\
IMC & 0,110 & 0,001 & 0,112 & $0,06-0,21$ & 0,108 & $0,04-0,14$ \\
PCR & 0,072 & 0,017 & 0,069 & $0,01-0,10$ & 0,067 & $0,01-0,16$
\end{tabular}

Modelo 1: ajustado por edad. Modelo 2: ajustado por edad, actividad física, tabaquismo.

Fuente: CRELES-RC. Año 2011

CUADRO 4

Costa Rica. Correlación univariada por estrato entre factores de riesgo de enfermedad cardiovascular en parejas participantes en el proyecto CRELES-RC. ( $n=1084$ parejas)

\begin{tabular}{lccccccccc}
\hline $\begin{array}{l}\text { Factor de } \\
\text { riesgo }\end{array}$ & Rural & Urbana & $\boldsymbol{q}$ & Otra & GAM & $\boldsymbol{q}$ & $\leq 6$ años & $>6$ años & $\boldsymbol{q}$ \\
\hline CT & 0,091 & 0,041 & 0,05 & 0,114 & $-0,019$ & 0,13 & 0,116 & 0,059 & 0,06 \\
C-HDL & 0,072 & 0,082 & 0,01 & 0,067 & 0,067 & 0,00 & 0,071 & 0,078 & 0,01 \\
HbA1C & 0,148 & 0,106 & 0,04 & 0,176 & 0,058 & 0,12 & 0,090 & 0,101 & 0,01 \\
PS & 0,042 & 0,136 & 0,09 & 0,082 & 0,089 & 0,01 & 0,061 & 0,077 & 0,02 \\
PD & 0,090 & 0,153 & 0,06 & 0,101 & 0,140 & 0,04 & 0,092 & 0,101 & 0,01 \\
CC & 0,040 & 0,107 & 0,07 & 0,092 & 0,052 & 0,04 & 0,070 & 0,010 & 0,06 \\
IMC & 0,069 & 0,102 & 0,03 & 0,110 & 0,066 & 0,04 & 0,108 & 0,133 & 0,03 \\
PCR & 0,093 & 0,126 & 0,03 & 0,073 & 0,166 & 0,09 & 0,063 & 0,114 & 0,05 \\
q: $q$ de Cohen & & & & & & & & &
\end{tabular}

Fuente: CRELES-RC. Año 2011

El modelo logístico univariado (modelo 1 ) indica que el OR de que el esposo tenga colesterol alto, bajo C-HDL, diabetes, hipertensión, AC, IMC superior al normal o inflamación dado que la esposa posee dicho factor riesgo fueron 1,230 (IC95\%: 0,96-1,57), 1,288 (IC95\%: 0,96-1,73); 2,713 (IC95\%: 1,94 - 3,79); 1,349 (IC95\%: 1,06 - 1,72); 1,434 (IC95\%: 1,03 - 1,99); 1,723 (IC95\%: 1,22 - 2,43) y 1,380 (IC95\%: 1,04 - 1,82), respectivamente. Además, el ajuste separado considerando factores de riesgo del esposo (modelo 2 ) y de la esposa (modelo 3 ) muestran OR similares a los obtenidos en el modelo 1 (Cuadro 5). Además, pudo verificarse que la asociación conyugal fue simétrica, es decir, el OR de que el esposo desarrolle un factor de riesgo considerando el estado de dicho factor en la esposa, fue similar al OR de que dicho factor de riesgo se desarrolle en la esposa tomando en cuenta 
el estado del factor en el esposo. Dada esta similitud, en el documento solo se muestran los resultados del modelo que considera como variable respuesta el factor de riesgo del esposo.

\section{CUADRO 5}

Costa Rica. Odds Ratios para factores de riesgo de enfermedad cardiovascular en parejas participantes en el proyecto CRELES-RC. ( $n=1084$ parejas)

\begin{tabular}{lcccccc}
\hline \multicolumn{1}{c}{$\begin{array}{c}\text { Factor de } \\
\text { riesgo }\end{array}$} & \multicolumn{2}{c}{ Modelo 1 } & \multicolumn{2}{c}{ Modelo 2 } & \multicolumn{2}{c}{ Modelo 3 } \\
& OR & IC 95\% & OR & IC 95\% & OR & IC 95\% \\
\hline Colesterol alto & 1,230 & $0,96-1,57$ & 1,211 & $0,95-1,55$ & 1,240 & $0,97-1,58$ \\
Bajo C-HDL & 1,288 & $0,96-1,73$ & 1,313 & $0,98-1,77$ & 1,289 & $0,96-1,74$ \\
Diabetes * & 2,713 & $1,94-3,79$ & 2,837 & $2,02-3,98$ & 2,731 & $1,95-3,83$ \\
Hipertensión * & 1,349 & $1,06-1,72$ & 1,357 & $1,06-1,74$ & 1,416 & $1,10-1,82$ \\
AC * & 1,434 & $1,03-1,99$ & 1,508 & $1,08-2,10$ & 1,477 & $1,06-2,05$ \\
IMC superior al normal* & 1,723 & $1,22-2,43$ & 1,777 & $1,25-2,53$ & 1,713 & $1,21-2,42$ \\
Inflamación * & 1,380 & $1,04-1,82$ & 1,357 & $1,02-1,80$ & 1,366 & $1,03-1,81$ \\
\hline
\end{tabular}

Modelo 1: ajuste univariado. Modelo 2: ajustado por edad, tabaquismo y actividad física del esposo. Modelo 3: ajustado por edad, tabaquismo y actividad física de la esposa. ${ }^{*} p<0,05$

Fuente: CRELES-RC. Año 2011

\section{DISCUSIÓN}

El estudio analizó las correlaciones presentes entre diversos factores de riesgo de enfermedad cardiovascular en 1084 parejas participantes en el proyecto CRELES-RC.

Los factores metabólicos que influyen en enfermedad cardiovascular considerados en el estudio mostraron correlaciones relativamente pequeñas, pero significativas, entre los cónyuges. Las correlaciones se mantienen luego de ajustar por edad y otras variables confusoras. Factores que pueden estar influenciados por componentes genéticos y ambientales como la presión sanguínea, la hemoglobina glicosilada y el colesterol se correlacionaron positivamente entre los cónyuges. En el caso de la PCR, recientemente relacionada con enfermedad cardiovascular (Ridker, Cushman, Stampfer, Tracy, \& Hennekens, 1997; Saleh et al., 2005; O'Doherty et al., 2014; Oikawa et al., 2019), también mostró correlación significativa $(p=0,017)$ entre las parejas.

En cuanto a la fuerza de la asociación, es importante mencionar que en el caso de la presión sanguínea se hallaron valores similares a los resultados de análisis univariados obtenidos en otros estudios (Jee et al., 2002; McAdams et al., 2011) que involucran a la población coreana y de algunas comunidades de Estados Unidos, respectivamente. También se hallaron similitudes con ajustes multivariados que involucraron varias poblaciones europeas (Di Castelnuovo et al., 2007). En el caso de la CC, se observaron valores similares a los presentados en la población coreana (Kim et al., 2006), al igual que en el caso del colesterol total (Jee et al., 2002). Para el caso de la PCR, si bien la correlación hallada es significativa, los valores obtenidos en la población costarricense son considerablemente menores a 0,17 presentado en otras regiones (Di Castelnuovo et al., 2011). Por otro lado, si bien la HbA1c mostró una correlación significa para la población de estudio, el valor es inferior al 0,55 hallado en Korea (Kim et al., 2006).

Finalmente, un metanálisis (Di Castelnuovo et al., 2009) estimó que la correlación entre los cónyuges es de 0,07 (IC95\%: 0,04; 0,10) para el colesterol total; 0,10 (IC95\%: -0,05; 0,24) para el colesterol HDL; 0,15 (IC95\%: -0,03; 0,33) para la glucosa en sangre; 0,10 (IC95\%: 0,03; 0,23) para la 
presión arterial sistólica; 0,09 (IC95\%: 0,00; 0,19) para la presión diastólica; 0,10 (IC95\%: -0,01; 0,21) para la CC y 0,15 (IC95\%: 0,05; 0,25) para el IMC. Todos los valores del estudio se encuentran dentro de los rangos establecidos en el metanálisis.

Por otro lado, en el caso de los esposos, los resultados de la regresión logística del estudio también sugieren que tener un cónyuge con un factor de riesgo (hipertensión, diabetes, adiposidad central, IMC superior al normal e inflamación) incrementa el OR $(1,349 ; 2,713 ; 1,434 ; 1,723$ y 1,380, respectivamente) de que el otro cónyuge tenga dicho factor de riesgo, incluso después de considerar el efecto de otros factores (edad, tabaquismo, actividad física). En el caso del colesterol (CT alto y bajo $C$-HDL) el incremento podría ser más modesto en dicha población $(p>0,05)$. Los resultados para las mujeres son similares, lo que indica que el medio ambiente ejerce un efecto similar en el esposo como en la esposa (McAdams et al., 2011). En el caso de la estimación de los OR, comportamientos similares han sido reportados para hipertensión (Hippisley-Cox, Coupland, Pringle, Crown, \& Hammersley, 2002; McAdams et al., 2011; Lee et al., 2011) para la CC (Kim et al., 2006; Lee et al., 2011). Para la diabetes, se obtuvo un aumento relevante en la probabilidad si se comparan con los valores de otros estudios (Kim et al., 2006; Hippisley-Cox et al., 2002; Lee et al., 2011). En el caso del colesterol alto, los valores del estudio son relativamente menores a 1,44 o 1,51 reportados para la hiperlipidemia (Hippisley-Cox et al., 2002). En el metanálisis, los OR para la concordancia de hipertensión fue 1,21 (IC95\%: 1,16-1,26), para diabetes 1,16 (IC95\%: 1,03-1,31) y para la obesidad 1,44 (IC95\%: 1,16-1,78) (Di Castelnuovo et al., 2009).

Dentro de las limitaciones del estudio, puede mencionarse que los análisis no contemplan datos sobre la duración del matrimonio o convivencia, así como de la calidad de dicha convivencia, ya que los estresores psicosociales están relacionados con un mayor riesgo de infarto agudo de miocardio (Rosengren et al., 2004), es decir, la angustia experimentada en la vida de pareja puede afectar el riesgo cardiovascular de los integrantes de la pareja. Al ser un estudio de corte transversal, no es posible detectar cambios en el comportamiento de los factores de riesgo en el transcurso de la convivencia conyugal y tampoco pueden establecerse relaciones causales.

Dentro de las principales conclusiones pueden mencionarse que el estudio reveló la existencia de concordancia conyugal significativa, aunque relativamente pequeña, en distintos factores de riesgo de enfermedad cardiovascular. Las correlaciones y los OR obtenidos para los distintos factores de riesgo de enfermedad cardiovascular en los cónyuges de la población costarricense en estudio no se alejan, en gran medida, de las relaciones encontradas en otras latitudes, por lo que se respalda la hipótesis de que un entorno compartido impacta en los diferentes factores de riesgo cardiovasculares. Estudios anteriores han mencionado que la concordancia presente en varios factores de riesgo cardiovascular se debe, principalmente, al resultado de un emparejamiento selectivo positivo (Hippisley-Cox et al., 2002), esto es, la tendencia de casarse con una persona de constitución y comportamientos similares (Di Castelnuovo et al., 2007). Finalmente, estos resultados pueden contribuir a la idea de que los profesionales de la salud pública puedan poner sus esfuerzos en la detección y el tratamiento de los factores de riesgo en los cónyuges como pareja y no tratarlos como personas separados.

\section{AGRADECIMIENTOS}

"Costa Rica: Estudio de longevidad y envejecimiento saludable (CRELES)" es un proyecto de investigación de la Universidad de Costa Rica, realizado por el Centro Centroamericano de Población (CCP) y de la Universidad de California-Berkeley, en colaboración con el Instituto. de Investigaciones en Salud, y los laboratorios del Hospital San Juan de Dios y la Oficina de Salud, UCR. Investigadores principales: Luis Rosero-Bixby y William H. Dow. Co-investigadores: Xinia Fernández y Gilbert Brenes-Camacho. Investigadores colaboradores: Ericka Méndez, Guido Pinto, Hannia Campos, Kenia 
Barrantes, Alberto Cubero, Fernando Coto, Pamela Jiménez, Jesús Granados y Yesi González. Personal de campo: José Solano, Julio Palma, Jenny Méndez, Maritza Aráuz, Mabelyn Gómez, Marcela Rodríguez, Geovanni Salas, Jorge Vindas, Katthya Alvarado, Randall González, Roberto Patiño, Carlos Robert y Jimmy Navarro.

\section{ÉTICA, CONFLICTO DE INTERESES Y DECLARACIÓN DE FINANCIAMIENTO}

Los autores declaran haber cumplido con todos los requisitos éticos y legales pertinentes, tanto durante el estudio como en el manuscrito; que no hay conflictos de interés de ningún tipo, y que todas las fuentes financieras se detallan plena y claramente en la sección de agradecimientos. Asimismo, están de acuerdo con la versión editada final del documento. El respectivo documento legal firmado se encuentra en los archivos de la revista.

La contribución de los autores es como se detalla a continuación: E.A.F.: investigador, análisis de datos y redacción del artículo. A.M.C.A.: investigadora y redacción del artículo.

\section{REFERENCIAS}

Aguilar, E., \& Carballo, A. (2020). Factores asociados a la Proteína C en la población costarricense nacida entre 1945 y 1955. Población y Salud en Mesoamérica, 17(2). DOI: 10.15517/psm.v17i2.39798.

American Diabetes Association, (2018). 2. Classification and diagnosis of diabetes: standards of medical care in diabetes. Diabetes Care 42(1), 13-28. DOI: 10.2337/dc18-S002

Cohen J. (1988). Statistical Power Analysis for the Behavioral Sciences. Second Edition. Hillsdate, NJ: LEA. Recuperado de http://www.utstat.toronto.edu/ brunner/oldclass/378f16/readings/CohenPower.pdf.

Cook, W. L., \& Kenny, D. A. (2005). The actor-partner interdependence model: a model of bidirectional effects in developmental studies. International Journal of Behavioral Development, 29(2), 101-109. DOI: $10.1080 / 01650250444000405$

Di Castelnuovo, A., Quacquaruccio, G., Arnout, J., Cappuccio, F. P., de Lorgeril, M., Dirckx, C., ... Venezia, A. (2007). Cardiovascular risk factors and global risk of fatal cardiovascular disease are positively correlated between partners of 802 married couples from different European countries: Report from the IMMIDIET project. Thrombosis and Haemostasis, 98(3), 648-655. DOI: 10.1160/TH07-01-0024

Di Castelnuovo, A., Quacquaruccio, G., Donati, M.B., de Gaetano, G., \& lacoviello, L. (2009). Spousal concordance for major coronary risk factors: a systematic review and meta-analysis. American Journal of Epidemiogy, 169(1), 1-8. DOI: 10.1093/aje/kwn234

Dow, W.H., Brenes, G., \& Rosero, L. (2013). CRELES: Costa Rican Longevity and Healthy Aging Study, Retirement Cohort. Methods, Wave 1. Berkeley, CA: Department of Demography, University of California, Berkeley. Recuperado de http://www.creles.berkeley.edu

Fitzpatrick, J., Gareau, A., Lafontaine, M. F., \& Gaudreau, P. (2016). How to Use the Actor-Partner Interdependence Model (APIM) To Estimate Different Dyadic Patterns in MPLUS: A Step-by-Step Tutorial. The Quantitative Methods for Psychology, 12(1), 74-86. DOI: 10.20982/tqmp.12.1.p074

Hippisley-Cox, J., Coupland, C., Pringle, M., Crown, N., \& Hammersley, V. (2002). Married couples' risk of same disease: cross sectional study. BMJ (Clinical research ed.), 325(7365), 636. DOI: 10.1136/bmj.325.7365.636

Jee, S.H., Suh, I, Won, S.Y., \& Kim, M. (2002). Familial correlation and heritability for cardiovascular risk factors. Yonsei Medical Journal, 43(2), 160-164. DOI: 10.3349/ymj.2002.43.2.160 
Jurj, A., Wen, W., Li, H., Zheng, W., Yang, G., Xiang, Y., ... Shu, X. (2006). Spousal correlations for lifestyle factors and selected diseases in Chinese couples. Annals of Epidemiology 16,(4), $285-291$. DOI: 10.1016/j.annepidem.2005.07.060

Kim, H., Kang, D., Choi, K., Nam, C., Thomas, G., \& Suh, I. (2006). Spousal concordance of metabolic syndrome in 3141 Korean couples: a nationwide survey. Annals of Epidemiology, 16(4), 292-298. DOI: 10.1016/j.annepidem.2005.07.052

Lee M.H., Kim H.C., Thomas G.N., Ahn S.V., Hur N.W., Choi D.P., \& Suh I. (2011). Familial concordance of metabolic syndrome in Korean population-Korean National Health and Nutrition Examination Survey 2005. Diabetes Research and Clinical Practice, 93(3), 430-436. DOI: 10.1016/j.diabres.2011.06.002

McAdams, M., Coresh, J., Woodward, M., Butler, K. R., Kao, W. H., Mosley, T. H., ... Anderson, C. A. (2011). Hypertension status, treatment, and control among spousal pairs in a middle-aged adult cohort. American journal of epidemiology, 174(7), 790-796. DOI: 10.1093/aje/kwr167

O'Doherty, M., Jørgensen, T., Borglykke, A., Brenner, H., Schöttker, B., Wilsgaard, T., . . Kee, F. (2014). Repeated measures of body mass index and C-reactive protein in relation to all-cause mortality and cardiovascular disease: Results from the consortium on health and ageing network of cohorts in Europe and the United States (CHANCES). European Journal of Epidemiology, 29(12), 887-897. DOI: 10.1007/s10654-014-9954-8

Oikawa, T., Sakata, Y., Nochioka, K., Miura, M., Abe R., Kasahara, S., ... Hiroaki Shimokawa, H. (2019). Association between temporal changes in C-reactive protein levels and prognosis in patients with previous myocardial infarction- A report from the CHART-2 Study. International Journal of Cardiology, 293, 17-24. DOI: 10.1016/j.ijcard.2019.07.022

Pearson, T., Mensah, G., Alexander, R., Anderson, J., Cannon, R., Criqui, M., ... Vinicor, F. (2003). Markers of inflammation and cardiovascular disease: application to clinical and public health practice: a statement for healthcare professionals from the Centers for Disease Control and Prevention and the American Heart Association. Circulation, 107(3), 499-511. DOI: 10.1161/01.CIR.0000052939.59093.45

Qureshi, A., Fareed, M., Suri, K., Kirmani, J., \& Divani, A. (2005). Cigarette smoking among spouses: another risk factor for stroke in women. Stroke, 36(9), 74-76. DOI: 10.1161/01.STR.0000177475.30281.7f

Ridker, P., Cushman, M., Stampfer, M., Tracy, R., \& Hennekens, C. (1997). Inflammation, aspirin, and the risk of cardiovascular disease in apparently healthy men. The New England Journal of Medicine, 336, 973-979. DOI: 10.1056/NEJM199704033361401

Rosengren A, Hawken S, Ounpuu S, Sliwa, K., Zubaid, M., Almahmeed, W., ... Yusuf, S. (2004). Association of psychosocial risk factors with risk of acute myocardial infarction in 11119 cases and 13648 controls from 52 countries (the INTERHEART study): case-control study. The Lancet, 364(9438), 953-962. Recuperado de: http://citeseerx.ist.psu.edu/viewdoc/download?doi=10.1.1.630.1236\&rep=rep1\&type=pdf

Saleh, N., Svane, B., Hansson, L., Jensen, J., Nilsson, T., Danielsson, O., \& Tornvall, P. (2005). Response of serum C-reactive protein to percutaneous coronary intervention has prognostic value. Clinical Chemistry, 51, 2124-2130. DOI: 10.1373/clinchem.2005.048082

Smith, S. Benjamin, E., Bonow, R., Braun, L., Creager, M., Franklin, B., ... Taubert, K. (2011). AHA/ACCF Secondary Prevention and Risk Reduction Therapy for Patients With Coronary and Other Atherosclerotic Vascular Disease: 2011 Update: A Guideline From the American Heart Association and American College of Cardiology Foundation Endorsed by the World Heart Federation and the Preventive Cardiovascular Nurses Association. Journal of the American College of Cardiology, 58(23), 2432-2446. DOI: 10.1016/j.jacc.2011.10.824

Stas, L., Kenny, D. A., Mayer, A., \& Loeys, T. (2018). Giving Dyadic Data Analysis Away: A User-Friendly App for ActorPartner Interdependence Models. Personal Relationships, 25(1), 103-119. DOI: 10.1111/pere.12230 